\title{
Differential Geometric Statement of Variational Equations for Abstract Fluids
}

\author{
A. Montesinos \\ Departamento de Geometría y Topología, Facultad de Matemáticas, Burjasot (Valencia), Spain
}

\begin{abstract}
A new global approach for the variational equations of fluids is given. The concept of prefluid is introduced, together with its variational equations and examples.
\end{abstract}

\section{Introduction}

The intrinsic description of perfect fluids in Newtonian space-time that appears mostly in the literature (see $[1,3]$ ) resembles the one used for elastic media: the study of a one-parameter (the time) family of diffeomorphisms. Besides its kinematic flavor, the limitation of this approach is evident for example in the case of general relativity, where there are no preferred spacelike slices for the parametrization of those diffeomorphisms.

For compressible fluids the situation is even worse, for in addition to the above relativistic remark, we are confronted with the heterogeneous role of the equation of continuity, which is to be imposed as a constraint. Also there are two classical pictures, Euler's and Lagrange's, with corresponding variational principles (variations on the velocities, variations on the initial positions, see [7] for discussion), that cast more confusion into the differential geometric core of the problem.

To avoid these shortcomings we introduce two innovations. The first one is to take fluids as what they seem to be, i.e. vector densities. The second refers to the variational equations and consists of taking variations that proceed from vector fields on the base space instead of vector fields tangent to the fibres.

Thus, let $\pi: E^{(r)} \rightarrow M^{n}$ be the bundle of $r$-vector densities on $M$, where $M$ could be a configuration space-time. Let $\pi_{\infty}: J^{\infty}(\pi) \rightarrow M$ be the bundle of $\infty$-jets of local cross-sections of $\pi$. Then an abstract fluid is a section of $\pi$. If a $\pi_{\infty}$-horizontal $n$-form $\lambda$ (the Lagrangian) is given on $J^{\infty}(\pi)$, it defines a functional on abstract fluids in the standard way. If $X$ is a vector field on $M$ with flow $\phi_{t}$, it induces a vector field on the bundle of frames $F(M)$ via the flow $\phi_{t *}$. This new vector field induces a vector field in each associated bundle, and in particular, a vector field $\tilde{X}$ on $E^{(r)}$ that induces the desired variation on abstract fluids.

We show that the variational principle that corresponds to such variations leads in the case of conservative fluids to Euler's equation plus the equation of continuity. Therefore, the latter becomes a consequence of the variational 
principle and no longer an external constraint. More precisely, our variational equations show that the equation of continuity holds except in the circumstance of cavitation, and this is a nice feature that lends more strength to our approach.

One could think therefore that fluids are vector densities with roots in the base space, whereas fields (in the physical sense) have their ground on some bundle over it. In fact, the usual image prescribes that the variation must be vertical, or at least that the variation vector field on $E$ must be $\pi$-projectable (really, these two types of variations are almost the same thing), but otherwise freely chosen. To show that perhaps this is not always the case, we study also another variational principle that appears sometimes in disguise in the literature. It is given by variations that commute with the divergence operator; equivalently, $\pi$-projectable vector fields that vary the divergence of a section as if it were a true fluid (in the sense we have described above). In other words, sections of $E^{(r)}$ that are not fluids, but their divergences are; in view of this we call those sections prefluids. We compute their variational equations and give three examples.

First, we prove that if we consider a classical fluid of noninteracting particles as a prefluid, its variational equations are simply the Hamilton-Jacobi equation (plus the equation of continuity).

Second, we show that Schrödinger's equation is the equation of a prefluid (of course, this is a wholly unorthodox derivation of that equation).

Third, Maxwell equations. We start from a Lagrangian built as usual, except that we drop the term $A \cdot j$ and do not assume that the electromagnetic field comes from a potential. Taking it as a prefluid (its divergence is the current, i.e. a fluid!), we obtain the correct Lorentz force plus the condition that the electromagnetic field must be not only a closed but also an exact form in any Lorentzian space-time.

\section{Preliminary Notations and Results (cf. [5])}

Let $\pi: E \rightarrow M^{n}$ be a fibre bundle with $n \geqq 1$ (all objects are $C^{\infty}$ ). We denote by $\Gamma(\pi)$, the set of local cross-sections of $\pi$. $J^{k}(\pi), 0 \leqq k \leqq \infty$, the manifold of $k$-jets of local cross-sections of $\pi$. Thus $J^{0}(\pi)=E$. $\pi_{h, k}: J^{k}(\pi) \rightarrow J^{h}(\pi)$ for $k \geqq h$, and $\pi_{k}: J^{k}(\pi) \rightarrow M$, the natural projections. $j^{k}(s)$, the $k$-jet of $s \in \Gamma(\pi) . V(M)$, the Lie algebra of vector fields on a manifold $M$.

Let $X \in V(M)$. Its horizontal lift or total vector field $X^{H} \in V\left(J^{\infty}(\pi)\right)$ is defined as follows. If $f \in C^{\infty}\left(J^{\infty}(\pi)\right), m \in M, s \in \Gamma(\pi)$, and $u=j^{\infty}(s)(m)$, then $X^{H}(f)(u)$ $=X\left(f \circ j^{\infty}(s)\right)(m)$. This map $V(M) \rightarrow V\left(J^{\infty}(\pi)\right)$ is $C^{\infty}(M)$-linear and a Lie algebra homomorphism.

Let $V_{\pi}(E) \subset V(E)$ be the subalgebra of $\pi$-projectable vector fields on $E$. Let $X \in V_{\pi}(E)$ and $\tilde{\phi}_{t}, \phi_{t}$ be the flows of $X$ and its projection $\pi(X)$, respectively. If $s \in \Gamma(\pi)$ is defined in a neighborhood of $m \in M$, then $s_{t}=\tilde{\phi}_{t} \circ S^{\circ} \phi_{-t}$ is also a local cross-section of $\pi$ on a neighborhood of $\phi_{t}(m)$. If $u=j^{k}(\mathrm{~s})(\mathrm{m})$, the tangent at $t=0$ to the curve $t \rightarrow j^{k}\left(s_{t}\right)\left(\phi_{t}(m)\right)$ depends only on $X$ and $u$, thus defining a vector $X^{k}(u)$. The map $u \rightarrow X^{k}(u)$ defines a vector field $X^{k} \in V\left(J^{k}(\pi)\right)$. The limit $X^{\infty} \in V\left(J^{\infty}(\pi)\right)$ of the $X^{k}$ when $k \rightarrow \infty$ is called the integrable vector field defined by the variation $X$. This map $V_{\pi}(E) \rightarrow V\left(J^{k}(\pi)\right), 0 \leqq k \leqq \infty$, is a Lie algebra homomorphism, and we have $\left[X^{\infty}, Y^{H}\right]=[\pi(X), Y]^{H}$ for every $Y \in V(M)$. 
Let $\mathscr{V}$ be the $\pi_{\infty}$-vertical subbundle of $T J^{\infty}(\pi)$. The horizontal subbundle, $\mathscr{H}$, is given by the horizontal lift of $T M$ and we have $T J^{\infty}(\pi)=\mathscr{V} \oplus \mathscr{H}$. Thus the algebra of differential forms on $J^{\infty}(\pi)$ becomes bigraded and we put $\omega \in H_{s}^{r}(\pi)$ if the $(r+s)$ form $\omega$ on $J^{\infty}(\pi)$ is $r$ times vertical and $s$ times horizontal. Thus $d=D+\partial$, where $D$ and $\partial$ are the horizontal and vertical differentials, respectively, and $D^{2}=\partial^{2}=D \partial$ $+\partial D=0$.

A form $\omega \in H_{n}^{1}(\pi)$ is called a source form if for each $u \in J^{\infty}(\pi), \omega(u)$ can be looked at as the pullback of a $(n+1)$-form on $\pi_{0, \infty}(u) \in E$. In other words, if $X \in T J^{\infty}(\pi)$ and $\left(\pi_{0, \infty}\right)_{*} X=0$, then $i(X) \omega=0$. A fundamental result of Takens [5] is the following.

Lemma. Each $\omega \in H_{n}^{1}(\pi)$ can be written uniquely as $\omega=\omega_{1}+\omega_{2}$, where $\omega_{1}$ is a source form and $\omega_{2} \in \operatorname{Im} D$.

\section{Densities}

In the following, $E=E^{(r)}=\left(\Lambda^{r} T M\right) \otimes\left(\Lambda^{n} T^{*} M\right)$, i.e. $E$ will be the bundle of $r$-contravariant skewsymmetric densities on $M$. We recall the concept of divergence [2]. Let $\kappa: E^{(r)} \rightarrow \Lambda^{n-r} T^{*} M$ be the bundle isomorphism given by

$$
\kappa(s)=\frac{1}{r !} s\left(\sigma^{i_{1}}, \ldots, \sigma^{i_{r}} ; e_{i_{1}}, \ldots, e_{i_{r}}, \ldots\right),
$$

where $s \in E^{(r)},\left\{e_{i}\right\}$ is a base of $T_{\pi(\mathrm{s})} M,\left\{\sigma^{i}\right\}$ its dual, and we sum over repeated indices. Then, if $s \in \Gamma(\pi), \operatorname{div} s=(-1)^{r}\left(\kappa^{-1} \circ d \circ \kappa\right)(s)$.

We can regard $\kappa$ as an $(n-r)$-form on $E$ since, if $s \in E$ and $X_{r+1}, \ldots, X_{n} \in T_{s} E$, we can put $\kappa(s)\left(X_{r+1}, \ldots, X_{n}\right)=\kappa(s)\left(\pi_{*} X_{r+1}, \ldots, \pi_{*} X_{n}\right)$; thus $\kappa \in H_{n-r}^{0}(\pi)$.

We also define the $(n-r+1)$-form Div on $J^{1}(\pi)$ by

$$
\operatorname{Div}\left(j^{1}(s)(m)\right)\left(X_{r}, \ldots, X_{n}\right)=(d \kappa(s))(m)\left(\pi_{1 *} X_{r}, \ldots, \pi_{1 *} X_{n}\right),
$$

or, equivalently, $\mathrm{Div}=D \kappa$.

We shall use the following subalgebras of $V_{\pi}(E)$ :

$$
\begin{gathered}
V_{v}(E)=\left\{X \in V_{\pi}(E) \mid \pi_{*} X=0\right\}, \\
V_{\kappa}(E)=\left\{X \in V_{\pi}(E) \mid \mathscr{L}_{X} \kappa=0\right\}, \\
V_{\text {div }}(E)=\left\{X \in V_{\pi}(E) \mid \mathscr{L}_{X^{1}} \operatorname{Div}=0\right\} .
\end{gathered}
$$

Now, if $X \in V(M)$, we denote by $\tilde{X} \in V_{\pi}(E)$ the vector field induced on $E$ via the tangent and cotangent map of the flow of $X$. Also, let $l: \Gamma(\pi) \rightarrow V_{v}(E)$ be the vertical injection. With these notations we have:

\section{Proposition.}

i) If $X \in V(M)$, then $\tilde{X} \in V_{\kappa}(E) \cap V_{\mathrm{div}}(E)$;

ii) if $X \in V_{\kappa}(E)$, then $X=\widetilde{\pi(X)}$; therefore, $V_{\pi}(E)=V_{\kappa}(E) \oplus V_{v}(E)$;

iii) let $X \in V_{\pi}(E)$; then $X \in V_{\mathrm{div}}(E)$ if and only if $X-\pi(X)=$ is for some $s \in \Gamma(\pi)$ such that $\operatorname{div} s=0$;

iv) if $X \in V(M)$ and $s \in \Gamma(\pi)$, then

$$
\left(X^{H}-\tilde{X}^{\infty}-\left(\imath \mathscr{L}_{X} s\right)^{\infty}\right) \circ j^{\infty}(s)=0 .
$$


Proof. Let $s \in E$ and $\tilde{\phi}_{t}, \phi_{t}$ be the flows of $X \in V_{\pi}(E)$ and $\pi(X)$, respectively. Then $\left(\mathscr{L}_{X} \kappa\right)(s)=\left.\frac{d}{d t}\right|_{t=0}\left(\tilde{\phi}_{t}^{*} \kappa\right)(s)$. But if $X_{r+1}, \ldots, X_{n} \in T_{s} E$, we have

$$
\begin{aligned}
\left(\tilde{\phi}_{t}^{*} \kappa\right)(s)\left(X_{r+1}, \ldots, X_{n}\right) & =\frac{1}{r !}\left(\tilde{\phi}_{t} s\right)\left(\sigma^{i_{1}}, \ldots, \sigma^{i_{r}} ; e_{i_{1}}, \ldots, e_{i_{r}}, \phi_{t *} \pi_{*} X_{r+1}, \ldots, \phi_{t *} \pi_{*} X_{n}\right) \\
& =\kappa\left(\phi_{-t}^{*} \tilde{\phi}_{t} s\right)\left(X_{r+1}, \ldots, X_{n}\right)
\end{aligned}
$$

where $\phi_{t}^{*}$ stands for the flow of $\widetilde{\pi(X)}$. Thus $\left(\tilde{\phi}_{t}^{*} \kappa\right)(s)=\kappa\left(\phi_{-t}^{*} \tilde{\phi}_{t} s\right)$. Since $\kappa$ is injective as a map, $\left(\mathscr{L}_{X} \kappa\right)(s)=0$ for all $s \in E$ if and only if $\tilde{\phi}_{t}=\phi_{t}^{*}$, that is if and only if $X=\widetilde{\pi(X)}$. This proves ii) and also that for each $X \in V(M)$ we have $X \in V_{\kappa}(E)$. Since for each $Y \in V_{\pi}(E)$ we have $\mathscr{L}_{Y^{\infty}} D=D \mathscr{L}_{Y^{\infty}}$, it follows that $\mathscr{L}_{\tilde{X}^{1}} \operatorname{Div}=\mathscr{L}_{\tilde{X}^{\infty}}$ Div $=\mathscr{L}_{\tilde{X}^{\infty}} D \kappa=D \mathscr{L}_{\tilde{X}^{\kappa}}=0$, and this is i).

Now, let $X \in V_{v}(E) \cap V_{\text {div }}(E)$. We have $\mathscr{L}_{X^{1}}$ Div $=D \mathscr{L}_{X} \kappa$. But if $\tilde{\phi}_{t}$ is the flow of $X$ and $u \in E$, then as before

$$
\left(\mathscr{L}_{X} \kappa\right)(u)=\left.\frac{d}{d t}\right|_{t=0} \kappa\left(\tilde{\phi}_{t} u\right)=\kappa\left(\left.\frac{d}{d t}\right|_{t=0} \tilde{\phi}_{t} u\right)=\kappa(X(u))
$$

under the identification of the tangent spaces to a fibre of $\pi$ with the fibre itself. Thus $\mathscr{L}_{X} \kappa=\kappa \circ X$, whence $D(\kappa \circ X)=0$. This is equivalent to say that for each $u \in \Gamma(\pi)$ we have $\operatorname{div}(X \circ u)=0$ (cf. [5, Remark 3.7]). If $r \geqq 1$, by using a coordinate expression it follows easily that the $\operatorname{map} X \circ u: M \rightarrow E$ cannot depend on $u$. In other words, $X=\imath s$ for some $s \in \Gamma(\pi)$ such that $\operatorname{div} s=0$, which is iii) having in mind that the case $r=0$ is trivial.

As for iv), we observe first that $X^{H}-\tilde{X}^{\infty}$ is vertical and commutes with all horizontal lifts of vector fields on $M$. Hence, it is determined by its action upon functions on $E$. So, let $f \in C^{\infty}(E)$ and $\phi_{t}, \tilde{\phi}_{t}$ be the flows of $X$ and $\widetilde{X}$, respectively. We have

$$
\begin{aligned}
\left(X^{H}-\tilde{X}^{\infty}\right)(f) \circ j^{\infty}(s) & =\left.\frac{\partial}{\partial t}\right|_{t=0} f \circ\left(s \circ \phi_{t}-\tilde{\phi}_{t} \circ s\right)=\left.\frac{\partial}{\partial t}\right|_{t=0} f \circ\left(s-\tilde{\phi}_{t} \circ S \circ \phi_{-t}\right) \circ \phi_{t} \\
& =-\left.\frac{\partial}{\partial t}\right|_{t=0} f \circ \tilde{\phi}_{t} \circ S \circ \phi_{-t}=\left(l \mathscr{L}_{X} s\right)(f),
\end{aligned}
$$

by the definition of $X$ and the Lie derivative, and our claim follows.

We shall need some technicalities about contractions. Let $\beta$ be a $p$-form on $M$ and $s$ a cross-section of $\pi: E^{(r)} \rightarrow M$. If $r \geqq p, \beta \cdot s$ will be the cross-section of $\pi: E^{(r-p)} \rightarrow M$ given by

$$
(\beta \cdot s)\left(\alpha^{p+1}, \ldots, \alpha^{r} ; X_{1}, \ldots, X_{n}\right)=\beta\left(e_{i_{1}}, \ldots, e_{i_{p}}\right) s\left(\sigma^{i_{1}}, \ldots, \sigma^{i_{p}}, \alpha^{p+1}, \ldots, \alpha^{r} ; X_{1}, \ldots, X_{n}\right)
$$

where $\left\{e_{i}\right\}$ is a local frame with dual $\left\{\sigma^{i}\right\}, \alpha^{p+1}, \ldots, \alpha^{r}$ are 1 -forms, $X_{1}, \ldots, X_{n}$ $\in V(M)$, and we sum over repeated indices.

If $r<p, \beta \cdot s$ will be the cross-section of $\Lambda^{p-r} T^{*} M \otimes \Lambda^{n} T^{*} M$ given by $(\beta \cdot s)\left(Y_{r+1}, \ldots, Y_{p} ; X_{1}, \ldots, X_{n}\right)=\beta\left(e_{i_{1}}, \ldots, e_{i_{r}}, Y_{r+1}, \ldots, Y_{p}\right) s\left(\sigma^{i_{1}}, \ldots, \sigma^{i_{r}} ; X_{1}, \ldots, X_{n}\right)$ 
We shall use the following formulae:

$$
\begin{gathered}
\operatorname{div}(\beta \cdot s)=(-1)^{p} \beta \cdot \operatorname{div} s+\frac{(-1)^{p+1}}{p+1} d \beta \cdot s, \quad r>p \\
\mathscr{L}_{X} s=-\operatorname{div}(X \wedge s)-X \wedge \operatorname{div} s, \quad X \in V(M), \\
\beta \cdot(X \wedge s)=(-1)^{p-1} p i(X)(\beta \cdot s), \quad p=r+1 .
\end{gathered}
$$

\section{Interpretation}

In the calculus of variations we must keep in mind that the variation could affect both the field $s$ and its domain of definition, and therefore, the domain of integration. For if $s$ is a local section of $E$ on $U \subset M, X \in V_{\pi}(E)$ and $\tilde{\phi}_{t}, \phi_{t}$ are the flows of $X$ and $\pi(X)$ respectively, then $s_{t}=\tilde{\phi}_{t} \circ S^{\circ} \phi_{-t}$ is the varied section and it is defined on $\phi_{t}(U)$. Thus, $\phi_{t}$ drags the section along $M$ and $\pi(X)$ measures the rate of this dragging. This vector field reflects itself on the image of $s$ as $s_{*} \circ \pi(X)$; then $X \circ s$ $-s_{*} \circ \pi(X)$ is a $\pi$-vertical vector field along $s$ and measures the rate of variation of $s$ as reckoned by an observer that remains fixed on $M$. In this sense, $V_{\pi}(E)$ can be regarded as the space of general or unrestricted variations. However, if we consider only first variations of the functional, the volume term depends only on the $\pi_{\infty}$-vertical vector field $\left(X^{\infty}-\pi(X)^{H}\right) \circ j^{\infty}(s)$, which is the extension to $J^{\infty}(\pi)$ of the vertical variation $X \circ s-S_{*} \circ \pi(X)$. Due to this, the interpretation of the choice of $V_{v}(E), V_{\kappa}(E), V_{\mathrm{div}}(E)$ as spaces of variations could be limited to the restrictions imposed upon $\left(X^{\infty}-\pi(X)^{H}\right) \circ j^{\infty}(s)$ by those choices. As always, these restrictions can be regarded as constraints upon the system represented by the section $s$.

\subsection{The Case $V_{v}(E)$}

Given $Y \in V_{\pi}(E)$, there is always some $X \in V_{v}(E)$ such that $\left(Y^{\infty}-\pi(Y)^{H}-X^{\infty}\right) \circ j^{\infty}(s)=0$, as it is easily proved. Hence, to take $V_{v}(E)$ or $V_{\pi}(E)$ as the space of variations is essentially the same thing. Thus, there is no restriction, the field $s$ has no constraints. Therefore, the fact that $E$ may be a tangent tensor bundle is irrelevant as far as the formal variational equations are concerned. We could say that $s$ represents a field in the usual physical sense, but not a fluid.

For instance, let $M=\mathbb{R}^{4}$ be the Minkowski space-time and $A$ a 1-form on $M$ representing the electromagnetic potential. The usual Lagrangian form for the electromagnetic field in absence of charges is given by $F^{2} \tau$, where $F=d A$, $F^{2}=F^{i j} F_{i j}$, and $\tau=d t \wedge d x \wedge d y \wedge d z$. Maxwell's equations are obtained from the condition $\delta \int F^{2} \tau=0$ for free variations $\delta A$. Thus, here $E=T^{*} M, s=A$, the Lagrangian form $\lambda=F^{2} \tau$ is a 4-form on $J^{1}(\pi)$ that belongs to $H_{4}^{0}(\pi)$, and the space of variations is $V_{v}(E)$. The fact that $E$ is the cotangent bundle is used explicitly only in the exterior differential of $A$, that is in the definition of $\lambda$. From this point the formal process of obtaining the variational equations is the same used for any bundle.

\subsection{The Case $V_{\kappa}(E)$}

If $X \in V_{\kappa}(E)$, then $X=\widetilde{\pi(X)}$ and $\left(X^{\infty}-\pi(X)^{H}\right) \circ j^{\infty}(s)=-\left(\imath \mathscr{L}_{\pi(X)} s\right)^{\infty} \circ j^{\infty}(s)$. Therefore, the variation takes place in $M$ rather than in $E$. To show in what sense 
we can say that $s$ is a fluid, let us consider that $r=1$. If $s \in \Gamma(\pi)$ and $s(m) \neq 0$ for all $m$ in an open subset $U \subset M$, we can interpret $s$ as a line field together with a density of lines. Indeed, the line field is given by the directions that are parallel to $s$, and if $i: B \rightarrow U$ is a hypersurface, where $i$ denotes the inclusion, $i^{*}(\kappa(s))$ is a volume form on $B$ which is nonzero if $B$ is transversal to the lines of $s$ and measures the flux or density of lines through $B$. Thus, let $X \in V_{\pi}(E)$ and $\tilde{\phi}_{t}, \phi_{t}$ be the flows of $X$ and $\pi(X)$. The varied section is $s_{t}=\tilde{\phi}_{t} \circ S^{\circ} \phi_{-t}$; then $s_{t}$ has its own lines of current, and the question is whether the line of $s_{t}$ passing through $\phi_{t}(m)$ is the variation of the line of $s$ passing through $m$, for any $m$ in $U$. In other words, whether integral curves are applied by the variation upon integral curves. This can be studied as follows: in the manifold $U \times \mathbb{R}$ we have the line field given at $(m, t)$ by $\left(s_{t}(m), 0\right)$, where we identify $s_{t}(m)$ with the direction determined by itself; we also have the "variation line field" $\left(\pi(X)\left(\phi_{t}(m)\right), \partial / \partial t\right)$. The condition is then that the plane field determined by both line fields should be integrable. After calculation we can express this as

$$
(X-\overline{\pi(X))} \circ s=(l f s) \circ s
$$

for some function $f$ on $U$.

As for the flux, let $\phi_{t} \circ i: B \rightarrow M$ be the varied hypersurface; the flux through it is given by $\left(\phi_{t} \circ i\right)^{*}\left(\kappa\left(s_{t}\right)\right)$. Then we can say that the variation is flux preserving if and only if $\frac{d}{d t}\left(\phi_{t} \circ i\right)^{*}\left(\kappa\left(s_{t}\right)\right)=(s \circ i)^{*} \mathscr{L}_{X} \kappa=0$. Since $\mathscr{L}_{X} \kappa=\mathscr{L}_{X-\widetilde{\pi(X)}} \kappa$ and $X-\widetilde{\pi(X)}$ $\in V_{v}(E)$, we have the condition $i^{*} \kappa((X-\widetilde{\pi(X)}) \circ s)=0$ (cf. Sect. 3), which together with (4) gives $i^{*} \kappa(f s)=0$. Thus, if $B$ is transverse to the lines of $s$, we have $\left(f_{s}\right) \circ i=0$. So, the variation carries lines into lines and preserves the flux if and only if $X \in V_{\kappa}(E)$. Hence, the choice of $V_{\kappa}(E)$ as the space of variations amounts to the constraint of preserving the "identity" of the lines of current and their density. We say that $s$ represents a fluid.

Example 1. Let $M=\mathbb{R} \times \mathbb{R}^{3}$ with coordinates $\left(x^{0}, x^{a}\right)$ represent the Newtonian space-time. We take coordinates $\left(x^{0}, x^{a}, y^{0}, y^{a}\right)$ for $E^{(1)}$ in such a manner that for a fluid with current $s=\left(s^{0} \partial / \partial x^{0}+s^{a} \partial / \partial x^{a}\right) \otimes \tau$, where $\tau=d x^{0} \wedge d x^{1} \wedge d x^{2} \wedge d x^{3}$, we put $y^{0}(s)=s^{0}, y^{a}(s)=s^{a}$. Then, if $s$ is the current of a compressible non-viscous fluid, its usual Lagrangian form is

$$
\lambda=\left(\frac{1}{2 y^{0}} y^{a} y^{a}-(V+e) y^{0}\right) \tau,
$$

where $V$ and $e$ are, respectively, the potential energy and the internal energy per unit of mass. The function $V$ can depend on $\left(x^{0}, x^{a}\right)$, and $e$ is supposed to depend only on the mass density $y^{0}$. Let $X=X^{0} \partial / \partial x^{0}+X^{a} \partial / \partial x^{a} \in V(M)$. Then $\tilde{X}=X^{\alpha} \partial / \partial x^{\alpha}+y^{\beta}\left(\partial X^{\alpha} / \partial x^{\beta}-\delta_{\beta}^{\alpha} \partial X^{\gamma} / \partial x^{\gamma}\right) \partial / \partial y^{\alpha}$, where the indices run from 0 to 3 .

Note that if we consider $\tau$ fixed, $s$ could be regarded as a vector field, and we would have the same coordinates $y^{\alpha}$ for $T M$. However, the extension of $X$ to $T M$ would be $\tilde{X}=X^{\alpha} \partial / \partial x^{\alpha}+y^{\beta}\left(\partial X^{\alpha} / \partial x^{\beta}\right) \partial / \partial y^{\alpha}$, that is the term $-y^{\alpha}\left(\partial X^{\gamma} / \partial x^{\gamma}\right) \partial / \partial y^{\alpha}$, that depends essentially on the divergence of $X$, would be missing. The choice of $E^{(1)}$ instead of $T M$ is thus essential in our approach. If we put $\varrho=s^{0}, \varrho v^{i}=s^{i}$, then

$$
\lambda=\left(\frac{1}{2} \varrho v^{2}-(V+e) \varrho\right) \tau,
$$

which is the usual expression for the Lagrangian form. 


\subsection{The Case $X \in V_{\text {div }}(E)$}

There is some $u \in \Gamma(\pi)$ with $\operatorname{div} u=0$ such that $X=\widetilde{\pi(X)}+u$. Then, the left-hand side of (4) is $(u u) \circ s$. Since $u$ is not necessarily a multiple of $s$ and $\kappa(u \circ \circ s) \neq 0$ in general, we conclude that neither the flux nor the lines of current are preserved by the variation. Therefore, $s$ is not a true fluid. However, since $\mathscr{L}_{X^{1}}$ Div $=0$, the variation preserves the flux and the lines of current of divs. In other words, sis not a true fluid but its divergence is; in this sense, we shall use the name prefluids for such entities.

Let us clear this up. If $X \in V_{\pi}(E)$, we put $\tilde{\phi}_{t}, \phi_{t}, \phi_{t}^{*}$ to denote the flows of $X, \pi(X)$ and $\overline{\pi(X)}$, respectively. If $s \in \Gamma(\pi)$ and $m \in M$, we have after computation

where $s_{t}=\tilde{\phi}_{t} \circ S \circ \phi_{-t}$.

$$
\left(\mathscr{L}_{X^{1}} \operatorname{Div}\right)\left(j^{1}(s)(m)\right)=(-1)^{r} \kappa\left(\mathscr{L}_{\pi(X)} \operatorname{div} s+\left.\frac{\partial}{\partial t}\right|_{t=0} \operatorname{div} s_{t}\right)(m),
$$

Now we define the map $p(X): J^{\infty}(\pi) \rightarrow T E^{(r-1)}$ as follows. If $u=j^{\infty}(s)(m)$, $p(X)(u)$ is the tangent at $t=0$ to the curve $t \rightarrow\left(\operatorname{div} s_{t}\right)\left(\phi_{t}(m)\right)$. In other terms, $p(X)$ is the variation induced by $X$ upon divs. By computation we get

$$
\begin{aligned}
p(X)\left(j^{\infty}(s)(m)\right)= & \overline{\pi(X)}((\operatorname{div} s)(m))+\left(L_{\left.\mathscr{L}_{\pi(X)} \operatorname{div} s\right)(m)}\right. \\
& +\left.\frac{\partial}{\partial t}\right|_{t=0}\left(\operatorname{div} s_{t}\right)(m),
\end{aligned}
$$

where $\overline{\pi(X)}$ denotes the extension of $\pi(X)$ to $E^{(r-1)}$. Then $p(X)\left(j^{\infty}(s)(m)\right)$ $=\widetilde{\pi(X)}((\operatorname{div} s)(m))$ if and only if $\mathscr{L}_{X^{1}} \operatorname{Div}=0$. If we put $(\operatorname{div} s)_{t}=\phi_{t}^{*} \circ \operatorname{div} s^{\circ} \phi_{-t}$, we have $\operatorname{div} s_{t}=(\operatorname{div} s)_{t}$ if and only if $\mathscr{L}_{X^{1}} \mathrm{Div}=0$. That is, divs is varied as a fluid if and only if $s$ is varied as a prefluid.

It is well known (cf. [7, p. 147]) that the first attempts to obtain Euler's equation from an Eulerian variational principle led to an irrotational velocity of the fluid when the entropy was supposed to be constant. Now it is clear, where the mistake was: the equation of continuity was regarded as a constraint and introduced in the Lagrangian by means of a Lagrange multiplier. This is equivalent to take variations that preserve the divergence, that is to choose $V_{\text {div }}(E)$ as space of variations. Thus, those attempts were considering the fluid as a prefluid.

\section{Variational Equations. Conservation Laws}

Let $\lambda \in H_{n}^{0}(\pi)$ be the Lagrangian form, $A \subset M$ be an orientable regular compact domain with boundary $\partial A, X \in V_{\pi}(E)$, and $s \in \Gamma(\pi)$. Then we consider the integral

$$
I(t)=\int_{\phi_{t}(A)} j^{\infty}\left(s_{t}\right)^{*} \lambda,
$$

where, as always, $s_{t}=\tilde{\phi}_{t} \circ S \circ \phi_{-t}$ and $\tilde{\phi}_{t}, \phi_{t}$ are the flows of $X$ and $\pi(X)$, respectively. By the change of variables formula,

$$
I(t)=\int_{A}\left(j^{\infty}\left(s_{t}\right) \circ \phi_{t}\right) * \lambda .
$$

Thus, the derivative of this integral with respect to $t$ at $t=0$ is

$$
J=\int_{A} j^{\infty}(s)^{*} \mathscr{L}_{X^{\infty}} \lambda=\int_{A} j^{\infty}(s)^{*} i\left(X^{\infty}\right) d \lambda+\int_{\partial A} j^{\infty}(s)^{*} i\left(X^{\infty}\right) \lambda .
$$


Since $d \lambda \in H_{n}^{1}(\pi)$, it decomposes as $d \lambda=\omega+D \alpha$, where $\omega$ is a uniquely defined source form and $\alpha \in H_{n-1}^{1}(\pi)$. Now, it is a trivial matter to prove the following slight generalization of $[5,3.7]$ :

Proposition. Let $\alpha \in H_{q}^{p}(\pi), s \in \Gamma(\pi)$, and $X_{1}, \ldots, X_{p}$ be integrable vector fields (not necessarily vertical). Then

$$
j^{\infty}(s)^{*}\left\{i\left(X_{1}\right) \ldots i\left(X_{p}\right) D \alpha\right\}=(-1)^{p} d j^{\infty}(s)^{*}\left(i\left(X_{1}\right) \ldots i\left(X_{p}\right) \alpha\right) .
$$

Also, since $\omega$ is a source form, $j^{\infty}(s)^{*} i\left(X^{\infty}\right) \omega=j^{\infty}(s)^{*} i\left(X^{\infty}-\pi(X)^{H}\right) \omega$. Therefore,

$$
J=\int_{A} j^{\infty}(s)^{*} i\left(X^{\infty}-\pi(X)^{H}\right) \omega+\int_{\partial A} j^{\infty}(s)^{*} i\left(X^{\infty}\right)(\lambda-\alpha) .
$$

Now, in order to have a well stated variational problem we must choose the subspace of $V_{\pi}(E)$ that specifies the type of variation. That is, we choose that subspace, impose that $X$ belongs to it and satisfies $X^{\infty} \circ j^{\infty}(s) \mid \partial A=0$, and then the condition that $J$ has to vanish for all such vector fields. The choice of the subspace $V_{v}(E)$ is the standard one and it leads to the necessary and sufficient condition $\omega \circ j^{\infty}(s)=0$, which is the well known Euler-Lagrange equation. Fluids are tied, however, to the variational subspace $V_{\kappa}(E)$. So, assume that in (5) we have $X=\widetilde{\pi(X)}$. Then

$$
j^{\infty}(s)^{*} i\left(X^{\infty}-\pi(X)^{H}\right) \omega=-\left(i\left({ }^{\prime} \mathscr{L}_{\pi(X)} s\right) \omega\right) \circ j^{\infty}(s),
$$

because $\omega$ is a source form.

It is obvious that there is a unique $r$-form $\omega_{s}$ on $M$ such that $\omega_{s} \cdot u$ $=(i(\imath u) \omega) \circ j^{\infty}(s)$ for all $u \in \Gamma(\pi)$. Then, by repeated application of (1), (2), (3) we have:

$$
\begin{aligned}
& j^{\infty}(s)^{*} i\left(X^{\infty}-\pi(X)^{H}\right) \omega=-\omega_{s} \cdot \mathscr{L}_{\pi(X)} s \\
& \quad=(-1)^{r} i(\pi(X))\left\{d \omega_{s} \cdot s-r\left(\omega_{s} \cdot \operatorname{div} s\right)\right\}+(-1)^{r+1} d \kappa\left(\omega_{s} \cdot(\pi(X) \wedge s)\right) .
\end{aligned}
$$

Thus

$$
\begin{aligned}
J= & (-1)^{r} \int_{A} i(\pi(X))\left\{d \omega_{s} \cdot s-r\left(\omega_{s} \cdot \operatorname{div} s\right)\right\} \\
& +\int_{\partial A} j^{\infty}(s)^{*} i\left(X^{\infty}\right)(\lambda-\alpha)+(-1)^{r+1} \kappa\left(\omega_{s} \cdot(\pi(X) \wedge s)\right),
\end{aligned}
$$

and therefore, we have

Theorem 1. Let $\lambda \in H_{n}^{0}(\pi), A \subset M$ be an orientable regular compact domain with boundary $\partial A$, and $s \in \Gamma(\pi)$. Then $I(t)$ is stationary at $t=0$ for all variations $X \in V_{\kappa}(E)$ with $X^{\infty} \circ j^{\infty}(s) \mid \partial A=0$ if and only if

$$
d \omega_{s} \cdot s-r\left(\omega_{s} \cdot \operatorname{div} s\right)=0
$$

in $A$.

Corollary 1. With the above assumptions, if $r=1$ (ordinary fluids) and s satisfies (6), then div $s=0$ in the open subset, where $\omega_{s} \cdot s \neq 0$ (equation of continuity).

Proof. It is enough to compute the contraction of (6) with a vector field parallel to $s$. 
Now we consider prefluids. So, let $X=\widetilde{\pi(X)}+\imath u$ with $\operatorname{div} u=0$ be a vector field in $V_{\text {div }}(E)$. We have as before

$$
\begin{aligned}
J= & \int_{A} \omega_{s} \cdot u+(-1)^{r} i(\pi(X))\left\{d \omega_{s} \cdot s-r\left(\omega_{s} \cdot \operatorname{div} s\right)\right\} \\
& +\int_{\partial A} j^{\infty}(s)^{*} i\left(X^{\infty}\right)(\lambda-\alpha)+(-1)^{r+1} \kappa\left(\omega_{s} \cdot(\pi(X) \wedge s)\right) .
\end{aligned}
$$

Then, if we take $\pi(X)=0$ and $u=\operatorname{div} K$ with $j^{\infty}(K) \mid \partial A=0$,

$$
\begin{aligned}
J & =\int_{A} \omega_{s} \cdot \operatorname{div} K=\int_{A}(-1)^{r} \operatorname{div}\left(\omega_{s} \cdot K\right)+\frac{1}{r+1} d \omega_{s} \cdot K \\
& =\frac{1}{r+1} \int_{A} d \omega_{s} \cdot K+(-1)^{r+1} \int_{\partial A} \kappa\left(\omega_{s} \cdot K\right)=\frac{1}{r+1} \int_{A} d \omega_{s} \cdot K .
\end{aligned}
$$

Therefore, a necessary condition for $J=0$ whenever $X \in V_{\text {div }}(E)$ with $X^{\infty} \circ j^{\infty}(s) \mid \partial A=0$ is $d \omega_{s}=0$. So, assume that $d \omega_{s}=0$ and take $X=\widetilde{\pi(X)}$. Then we obtain the necessary condition $\omega_{s} \cdot \operatorname{div} s=0$. Then, assuming that $d \omega_{s}=0$ and $\omega_{s} \cdot \operatorname{div} s=0$, the condition becomes

$$
J=\int_{A} \omega_{s} \cdot u=0
$$

whenever $\operatorname{div} u=0$ and $j^{\infty}(u) \mid \partial A=0$. Since $\omega_{s}$ is closed, we get by Poincaré duality that $\omega_{s}$ must be exact. Thus we have proved

Theorem 2. Let $\lambda \in H_{n}^{0}(\pi), A \subset M$ be an orientable regular compact domain with boundary $\partial A$ and $s \in \Gamma(\pi)$. Then $I(t)$ is stationary at $t=0$ for all variations $X \in V_{\mathrm{div}}(E)$ with $X^{\infty} \circ j^{\infty}(s) \mid \partial A=0$ if and only if

in $A$.

$$
\omega_{s} \cdot \operatorname{div} s=0 \text { and } \omega_{s} \text { is exact }
$$

Corollary 2. With the above assumptions, if $r=1$ and satisfies (7), then $\operatorname{div} s=0$ in the open subset, where $\omega_{\mathrm{s}} \neq 0$ (equation of continuity).

Let us assume that $\omega_{s}$ is exact, say $\omega_{s}=d \theta_{s}$, and that $\omega_{s} \cdot \operatorname{div} s=0$. Then for each $X \in V_{\text {div }}(E), X=\widetilde{\pi(X)}+u$, we have

$$
J=\int_{\partial A} j^{\infty}(s)^{*} i\left(X^{\infty}\right)(\lambda-\alpha)+(-1)^{r+1} \kappa\left(r \theta_{s} \cdot u+d \theta_{s} \cdot(\pi(X) \wedge s)\right) .
$$

The $(n-1)$-form under the integral sign can be considered as the momentum associated with the vector field $X$. This, and the corresponding expression for fluids, leads immediately to the following conservation laws.

Theorem 3. Let $\lambda \in H_{n}^{0}(\pi), s \in \Gamma(\pi)$ be a cross-section satisfying the condition (6) (respectively (7)), $X \in V_{\kappa}(E)$ (respectively $X \in V_{\text {div }}(E)$ ) be such that $\mathscr{L}_{X^{\infty}} \lambda=D \beta$ for some $\beta \in H_{n-1}^{0}(\pi)$, and $A \subset M$ be an orientable regular compact domain with boundary $\partial A$. Then

$$
\int_{\partial A} j^{\infty}(s)^{*}\left(i\left(X^{\infty}\right)(\lambda-\alpha)-\beta\right)+(-1)^{r+1}\left(\omega_{s} \cdot(\pi(X) \wedge s)\right)=0
$$

(respectively

$$
\int_{\partial A} j^{\infty}(s)^{*}\left(i\left(X^{\infty}\right)(\lambda-\alpha)-\beta\right)+(-1)^{r+1} \kappa\left(r \theta_{s} \cdot u+d \theta_{s} \cdot(\pi(X) \wedge s)\right)=0
$$

where $X=\overline{\pi(X)}+\imath u$ and $\left.\omega_{s}=d \theta_{s}\right)$. 


\section{Examples}

In order to make sure that our first variational principle works correctly for the usual non-viscous fluids, we take the Lagrangian form of Example 1. The standard formula for the Euler-Lagrange variational equations gives

$$
\omega=\left\{-\left(\frac{y^{a} y^{a}}{2\left(y^{0}\right)^{2}}+V+e+\frac{p}{y^{0}}\right) d y^{0}+\frac{y^{a}}{y^{0}}\right\} d y^{a} \wedge \tau,
$$

where $p=\left(y^{0}\right)^{2} \frac{d e}{d y^{0}}$ is the pressure (see [7]). If $s=\varrho\left(\partial / \partial x^{0}+v^{a} \partial / \partial x^{a}\right) \otimes \tau$, we get after putting $t=x^{0}$ :

$$
\omega_{s}=-\left(\frac{v^{2}}{2}+V+e+\frac{p}{\varrho}\right) d t+v^{a} d x^{a}
$$

Then Eq. (6) splits into

$$
\begin{gathered}
\varrho \frac{d v}{d t}+\varrho \operatorname{grad} V+\operatorname{grad} p=\left(\frac{\partial \varrho}{\partial t}+\operatorname{div}_{0}(\varrho v)\right) v, \\
\frac{1}{2} \varrho \frac{d v^{2}}{d t}+v \cdot(\varrho \operatorname{grad} V+\operatorname{grad} p)=\left(\frac{v^{2}}{2}+V+e+\frac{p}{\varrho}\right)\left(\frac{\partial \varrho}{\partial t}+\operatorname{div}_{0}(\varrho v)\right),
\end{gathered}
$$

where grad, $\operatorname{div}_{0}$, and $\frac{d}{d t}$ are the spatial gradient, the spatial divergence and the Eulerian derivative, respectively. If we multiply (8) by $v$ and subtract from (9) we get the following equation of compatibility (cf. Corollary 1)

$$
\left(\frac{1}{2} \varrho v^{2}-p-\varrho(V+e)\right)\left(\frac{\partial \varrho}{\partial t}+\operatorname{div}_{0}(\varrho v)\right)=0 .
$$

Thus, the equation of continuity holds in the points, where

$$
\frac{1}{2} \varrho v^{2} \neq p+\varrho(V+e) .
$$

In other words, the kinetic energy of a fluid cannot reach the sum of the energies given by the potential, the internal energy and the pressure; otherwise, the fluid may not satisfy $\frac{\partial \varrho}{\partial t}+\operatorname{div}_{0}(\varrho v)=0$, i.e. it could cavitate. This is a nice byproduct of our approach. If the equation of continuity holds, then (8) and (9) become

$$
\varrho \frac{d v}{d t}+\varrho \operatorname{grad} V+\operatorname{grad} p=0, \quad \frac{1}{2} \varrho \frac{d v^{2}}{d t}+v \cdot(\varrho \operatorname{grad} V+\operatorname{grad} p)=0,
$$

which are the usual Euler equations for the momentum and the energy.

Example 2. Now, we shall point out the relation existing between prefluids and Hamilton-Jacobi equation. Let $M=\mathbb{R} \times Q$, where $Q$ is any configuration space and $\mathbb{R}$ represents time. We consider a particle as a section of the bundle $M \rightarrow \mathbb{R}$. Let $\left(q^{a}\right),\left(q^{a}, v^{a}\right),(t)$ denote coordinates in $Q, T Q$, and $\mathbb{R}$, respectively, and $L=L\left(t, q^{a}, v^{a}\right)$ be a function on $\mathbb{R} \times T Q$, the Lagrangian of a particle. We want to define the Lagrangian form of a fluid constituted by non-interesting particles, each subject to 
that Lagrangian. To do that, we first make homogeneous $L$ by defining a new function on $(T M)^{0} \equiv(T \mathbb{R}-\{0\}) \times T Q$. We put $L^{\prime}=L^{\circ} \eta$, where $\eta:(T M)^{0} \rightarrow \mathbb{R}$ $\times T Q$ is given by $\eta\left(t, v^{0}, q^{a}, v^{a}\right)=\left(t, q^{a}, \frac{v^{a}}{v^{0}}\right)$. Note that $L^{\prime}$ depends on the coordinates of $M$ and on the direction of the world-line $\left(v^{0}, v^{a}\right)$, but not on its length. So, we multiply $L^{\prime}$ by an $n$-form on $M$ that we can write $y^{0} d t \wedge d q^{1} \wedge \ldots \wedge d q^{n-1}$ and obtain the Lagrangian form

$$
\lambda=y^{0} L^{\prime}\left(t, v^{0}, q^{a}, v^{a}\right) d t \wedge d q^{1} \wedge \ldots \wedge d q^{n-1} .
$$

By the homogeneity of $L^{\prime}$, the change of coordinates $y^{a}=\frac{v^{a}}{v^{0}} y^{0}$ gives

$$
\lambda=y^{0} L^{\prime}\left(t, y^{0}, q^{a}, y^{a}\right) d t \wedge d q^{1} \wedge \ldots \wedge d q^{n-1},
$$

where $\left(y^{0} \partial / \partial t+y^{a} \partial / \partial q^{a}\right) \otimes\left(d t \wedge d q^{1} \wedge \ldots \wedge d q^{n-1}\right)$ is the vector density describing the fluid. Note that in the points of $(T M)^{0}$, where $v^{0}=1$, that is in the image of the usual inclusion $\mathbb{R} \times T Q \rightarrow(T M)^{0}$ we have $y^{a}=y^{0} v^{a}$. This shows that $\left(y^{0}, y^{a}\right)$ can be interpreted as the current and $y^{0}$ as the density. If

$$
s=\left(s^{0} \partial / \partial t+s^{a} \partial / \partial q^{a}\right) \otimes\left(d t \wedge d q^{1} \wedge \ldots \wedge d q^{n-1}\right) \in \Gamma(\pi),
$$

we have with a slight abuse of notation

$$
\omega_{s}=\left(L \circ S-\left(\frac{\partial L}{\partial v^{a}} \circ S\right) \frac{s^{a}}{s^{0}}\right) d t+\left(\frac{\partial L}{\partial v^{a}} \circ s\right) d q^{a} .
$$

Now, if we consider $\lambda$ as the Lagrangian form of a prefluid, the equations (7) are

$$
\begin{gathered}
\frac{\partial L}{\partial v^{a}}=\frac{\partial S}{\partial q^{a}}, \quad h_{L}=-\frac{\partial S}{\partial t}, \\
\operatorname{div} s=0,
\end{gathered}
$$

where $h_{L}=-L+v^{a} \frac{\partial L}{\partial v^{a}}$ is the Hamiltonian. Equations (10) are equivalent to the Hamilton-Jacobi equation for the Lagrangian $L[6$, p. 91] and are complemented in our scheme with the equation of continuity (11).

Example 3. We shall show that the Schrödinger equation can be looked at as the variational equation of a prefluid. We start with the usual equation

$$
i h \frac{\partial \Psi}{\partial t}=-\frac{h^{2}}{2 m} \Delta \Psi+V \Psi
$$

If we put $\Psi=m^{-1 / 2} \mu^{1 / 2} \exp \left(\frac{i m}{h} \theta\right)$, where $\mu$ and $\theta$ are real functions, (12) decomposes into

$$
\begin{gathered}
\frac{\partial \mu}{\partial t}+\operatorname{div}_{0}(\mu \operatorname{grad} \theta)=0 \\
\Delta \mu=\frac{4 m^{2}}{h^{2}}\left(\frac{h^{2}}{8 m^{2}} \frac{(\operatorname{grad} \mu)^{2}}{\mu}+\frac{1}{2} \mu(\operatorname{grad} \theta)^{2}+\frac{V}{m} \mu+\frac{\partial \theta}{\partial t} \mu\right) .
\end{gathered}
$$


Now, (13) can be looked at as an equation of continuity for a fluid with density $\mu$ and irrotational velocity $v=\operatorname{grad} \theta$. The universal character of the equation of continuity for the case $r=1$ (cf. Corollaries 1 and 2) raises thus the question whether (13) and (14) could be obtained via our second variational principle. The answer is affirmative. In fact, let as before $\left(t, x^{a}\right)$ be the canonical coordinates in the space-time $\mathbb{R} \times \mathbb{R}^{3}$, and $\left(t, x^{a}, y^{0}, y^{a}\right)$ be the corresponding coordinates for $E^{(1)}$. We apply Theorem 2 to a prefluid with Lagrangian form

$$
\lambda=\left(\frac{1}{2} \frac{y^{a} y^{a}}{y^{0}}-\frac{V}{m} y^{0}-\frac{h^{2}}{8 m^{2}} \frac{y_{a}^{0} y_{a}^{0}}{y^{0}}\right) \tau
$$

where $\left(t, x^{a}, y^{0}, y^{a}, y_{0}^{0}, y_{a}^{0}, y_{0}^{a}, y_{b}^{a}\right)$ are the induced coordinates on $J^{1}(E)$. Then, if

$$
s=\mu\left(\frac{\partial}{\partial t}+v^{a} \frac{\partial}{\partial x^{a}}\right) \otimes \tau \in \Gamma(\pi)
$$

we obtain

$$
\omega_{s}=\left(\frac{h^{2}}{4 m^{2}} \frac{\Delta \mu}{\mu}-\frac{h^{2}}{8 m^{2}} \frac{(\operatorname{grad} \mu)^{2}}{\mu^{2}}-\frac{1}{2} v^{2}-\frac{V}{m}\right) d t+v^{a} d x^{a} .
$$

By Theorem 2, $\omega_{s}$ must be exact and $\omega_{s} \cdot \operatorname{div} s=0$. Hence $\omega_{s}=d \theta$, which is (14), and $\operatorname{div} s=0$ at the points, where $\omega_{s} \neq 0$, which is (13).

A similar Lagrangian has been used in [4] for a stochastic deduction of the Schrödinger equation.

Example 4. Our last example refers to $E^{(2)}$. Let $(M, g)$ be an orientable pseudoriemannian manifold, for instance a Lorentzian space-time. If $H \in \Gamma(\pi)$ (therefore, $H$ is a bivector density and may represent the electromagnetic field density) and $\tau$ is the Riemannian volume element, we can build the 2 -form $F$ by putting $F^{\#} \otimes \tau=H$, where $F^{\#}$ is the tensor field obtained by raising the indices of $F$ by means of $g$. Then $F \cdot H$ is an $n$-form on $M$, and if $F_{i j}$ are the components of $F$ in a local frame, then $F \cdot H=F^{i j} F_{i j} \tau$. Also, we put $j=\operatorname{div} H$ and define $u \in V(M)$ by $u \otimes \tau=j$. Let $0 \neq b \in \mathbb{R}$; the map $H \rightarrow b g(u, u)^{1 / 2} \tau+F \cdot H$ can be considered as a Lagrangian form on $J^{1}(E)$. Its first term represents the inertial energy of the current $u$; thus, it could be thought of as the energy of a mass current with a restmass density $b$ times the absolute value of the charge density of the electric current. Our Lagrangian differs from the traditional one in that the term $A \cdot j$, where $A$ would stand for the electromagnetic potential, is missing. But this is consistent, because the energy of a system of charges can be attributed to the electromagnetic field only, as it is well known.

After computation we find that $\omega_{H}=2 F+\frac{1}{2} b d \eta$, where $\eta=g(u, u)^{-1 / 2} g(u$, ). It must be remarked that in the computation we do not assume that $F$ is derived from a potential. If we consider $H$ as a prefluid, we have the variational equations:

$$
\begin{gathered}
F \text { is exact, that is } F=d A, \\
F(u, \quad)=-\frac{b}{4}(d \eta)(u, \quad) .
\end{gathered}
$$


If we denote by $v$ the unit vector field $g(u, u)^{-1 / 2} u$ that represents the velocity of the current, the last formula can be written in the form

$$
g\left(\nabla_{u} v,\right)=-\frac{4}{b} F(u,) .
$$

That is, we have the complete set of Maxwell equations together with the Lorentz force and the charge conservation. In fact, (15) expresses the existence of a global electromagnetic potential; $j=\operatorname{div} H$ as usual, except that here $j$ appears as the definition of the electric current density; $\operatorname{div} j=\operatorname{div} \operatorname{div} H \equiv 0$ expresses the conservation of charge; finally, (17) is the usual Lorentz force upon a charged fluid with rest-mass density $b$ times the absolute value of its charge density.

\section{References}

1. Arnold, V.: Sur la géometrie différentielle des groupes de Lie de dimension infinie et ses applications a l'hydrodynamique des fluids parfaits. Ann. Inst. Fourier Grenoble 16, 1, 319-361 (1966)

2. Greub, W., Halperin, S., Vanstone, R.: Connections, curvature, and cohomology, Vol. I. New York: Academic Press 1972

3. Marsden, J.: Applications of global analysis in mathematical physics. Boston: Publish or Perish Inc. 1974

4. Santos, E.: Sobre la deducción de la ecuación de Schrödinger a partir de la Mecánica Clásica. An. Física 68, 137-146 (1972)

5. Takens, F.: A global version of the inverse problem of the calculus of variations. J. Diff. Geom. 14, 543-562 (1979)

6. Woodhouse, N.: Geometric quantization. Oxford: Clarendon Press 1980

7. Yourgrau, W., Mandelstam, S.: Variational principles in dynamics and quantum theory. New York: Dover 1979

Communicated by H. Araki

Received November 26, 1983; in revised form April 20, 1984 
